\title{
The Effects of Synesthesia on Recall Memory Abilities in Teenagers
}

\author{
Emily Borr ${ }^{1}$
}

${ }^{1}$ Wekiva High School, Apopka, Florida, USA

\section{ABSTRACT}

Synesthesia is a rare phenomenon in which people associate an inducer, such as a sound, song, or sequence of time, with a specific concurrent, such as a color, shape, or physical sensation. Very little is known about what causes this phenomenon, although due to a recent influx of research, it has been recorded that synesthesia provides a wide array of cognitive benefits to those who experience it, with the most notable being memory. Children with synesthesia in group studies have not been shown to have advantageous memory, while adults with synesthesia in group studies have shown the opposite. However, a group study of this nature has never been conducted on teenagers with synesthesia, despite this being the age range where advantageous memory would seem likely to manifest. This study conducts an experimental analysis on the relationship between synesthesia and advantageous memory in teenagers for immediate, delayed, and matrix recall memory. The results of this study show that teenagers with synesthesia have a memory advantage in matrix recall memory, leading to the conclusion that, for some types of memory, synesthesia begins to provide a benefit in the years of adolescence.

\section{Introduction and Literature Review}

Synesthesia is an associative phenomenon in which normal sensory stimuli elicit a second perceptual response. The name "synesthesia" literally means "mixing of the senses." For example, in grapheme-color synesthesia, people may perceive the number 1 as "red" or the letter M as "blue" in their head. In sound-color synesthesia, people experience different colors for pitches, songs, instrument tambours, chords, etc. Those with sequence-space synesthesia experience groups of time, such as days of the week or months of the year, in specific sequences in the space around their body (e.g, January may be two feet in front to the left). Conventionally, the stimulus that causes a synesthetic experience (such as a number, letter, or song) is called the inducer, the elicited experience (such as a color) is called the concurrent, and a person with synesthesia is called a synesthete.

Synesthesia is a rare phenomenon, only occurring in 1-4\% of the population (Novich et al., 2011). Although there are many types of synesthesia, most of them have not yet been researched thoroughly enough to provide a proper analysis of their effects on the people with these types of synesthesia. To date, the best studied type of synesthesia is grapheme-color, described above as a number or letter triggering a color experience (Rothen et al, 2012).

Despite an influx of research on this phenomenon in the recent decade, very little is known about what exactly causes synesthesia. Hypotheses have been made that it may be learned through association at a young age (Winthoft $\&$ Winawer, 2013), or that synesthetes have increased hyperconnectivity globally and locally in the fusiform gyrus and inferior parietal lobe of the brain, which has shown to be true through MRI scanning (Hanggi et al, 2011). The only proven, widely-acknowledged information about synesthesia is that it is not a disease or disorder (Rothen et al, 2012), and that the experiences it induces are constant. In fact, the most reliable and widely used test for confirmation of synesthesia, the Synesthesia Battery (Eagleman et al, 2007), tests for consistency of concurrents over time to discern whether an individual is a synesthete or not. 
Novich, Cheng, and Eaglemen (2011) found that there are five distinct groupings of the various types of synesthesia: colored sequences, colored music, colored sensations, non-visual sequelae, and sequence space synesthesia. Of these, colored sequences, colored music, and sequence space synesthesia are among the most well studied types and can be described as a grapheme (letter or number) or sequence triggering a color, a sound triggering a color, or a sequence of time triggering a formation in the space around the synesthete, respectively. Colored sensations, similar to the other colored clusters, elicit colored concurrents, but have touch, pain, emotion, taste, or smell inducers instead of music, grapheme, or sequence inducers. Lastly, non-visual sequelae synesthesia is when a sound or vision triggers a smell, physical sensation, or taste.

\section{Synesthesia and Cognitive Benefits}

In consideration that synesthetes may have increased brain hyperconnectivity (Hanggi et al, 2011), it can be suggested that this difference in brain function would affect how synesthetes operate. Meier and Rothen (2013) found that color synesthetes do, indeed, have a unique cognitive style. Using the Verbalizer-Visualizer-Questionnaire on graphemecolor, sound-color, lexical-gustatory (in which words elicit tastes), and sequence-space synesthetes, they were able to conclude that the presence of a color synesthesia indicated high vivid imagery and visual style cognitive styles, suggesting that synesthetes learn and process better with verbal or visual information, depending on their synesthesia type. These findings imply that synesthetes may, in fact, have cognitive advantages where non-synesthetes do not.

Proof of this can be seen in a study done by Rouw and Scholte (2016), in which a group of synesthetes identified by MRI scanning were compared in general intelligence against a randomly selected portion of the Dutch population. Synesthetes were ages 20-26 and had a variety of synesthesia types that were first identified by a detailed questionnaire created by the researchers. The control population was taken from a recruitment center in the Netherlands, to ensure that it was an accurate representation of the general Dutch population. After taking the Intelligence Structure Test, synesthetes of all types were discovered to have better scores overall than the controls. Rouw and Scholte credited these findings to the idea that synesthesia provides an intelligence advantage that is not driven by a specific type of synesthesia or specific stimuli, but rather by the "general effect" of synesthesia.

Another recently discovered cognitive advantage is increased mental rotation skills in synesthetes whose concurrents appear in space and not in their mind's eye. In an experiment comparing synesthetes to control subjects in tasks measuring memory aptitude and rotation recall, the synesthetes significantly outperformed the controls. These results suggest that sequence-space synesthetes have better recall and mental-rotation skills due to their synesthesia allowing them to view sequences in space, giving them the overall advantage in spacial processing ability (Hale et al, 2014).

Chun and Hupé (2015) found a wide array of cognitive benefits in their synesthetes. After confirming their participants synesthesia with a test very similar to the Synesthesia Battery, they administered multiple tests (The Remote Associates Test, Visual Associates Test, Alternative Uses Test, Torrance Test for Creative Thinking: Visual, Wechsler Adult Intelligence Scale- Third Edition, and the Association of Meaning Test) that assessed verbal and visual convergent creativity, verbal and visual divergent creativity, verbal fluency, general cognition, and delayed and immediate recall memory. The researchers discovered increased verbal comprehension, visual convergent thinking, originality of verbal divergent thinking, usage of mental imagery, general knowledge, and vocabulary in synesthetes over controls, which could potentially be contributed to the "general effect" of synesthesia, as discovered by Rouw and Scholte (2016).

\section{Synesthesia and Memory}

Perhaps the most extensively studied benefit from synesthesia is improved memory abilities. The synesthesia-memory correlation has only recently gained definitive research, but the phenomenon itself has been examined since the 1960s. The first synesthete with well-documented extraordinary memory was Solomon Shereshevsky (Luria, 1968), who was able to memorize series of 50 numbers in three minutes, perfectly recall all 50 numbers immediately after 
memorization, and perfectly recall them again three years later. Other examples of synesthetes documented for extraordinary memory include Smilek's C (2002) and Robinson's M.P. (2015). These individual case studies were extremely influential in the early days of synesthesia research because, though they lack concrete confirmation, they reinforce the premise that the synesthesia-memory advantage reasonably exists.

However, most, if not all, of the synesthetes used in these individual case studies were selected for research because they were known to have extraordinary memory. In other words, these synesthetes, who were previously established to have better memory than the average population, are not an accurate representation of the majority of those with synesthesia, because most people, synesthete or not, do not present exceptional memory that is deemed worthy of further study. Therefore, these results may not be true for the general synesthete population and the phenomenon should instead be investigated with a group that more accurately represents average synesthetes.

Rothen and Meier (2009) did just this with a group of 13 young-adult grapheme-color synesthetes, but made the contradictory conclusion that synesthesia does not provide a memory advantage in the general synesthete population. For the memory task, participants and age- and education-matched controls were presented a task in which they were shown matrices of 50 numbers for three minutes and were asked to recall as many as they could after the allotted time. This task was conducted in three trials: one with all-black numbers, one with numbers in colors congruent to each synesthete's concurrents (e.g, if 1 was "red," 1 was presented in red), and one with colors incongruent to each synesthetes concurrents (e.g, if 1 was "red," 1 was presented in a color other than red). The results showed no general performance benefit for synesthetes over controls, contradicting the results of the previous single case studies. However, Rothen and Meier do also mention that, when the results were calculated per individual rather than per group, some synesthetes did show a performance advantage over controls. The researchers attribute this to the idea that some synesthetes have stronger associations than others, giving them stronger recall abilities.

Radvansky and Gibson (2011) found opposing results to Rothen and Meier (2009), despite using a very similar test in addition to the Deese-Roediger-McDermott False Memory Test with 10 college-aged synesthetes and matching controls. Gross and Neargarder (2011) also concluded that synesthesia provided a memory advantage in young-adult synesthetes over controls when they performed similar tests. Pfiefer et al (2014), Lunke and Meier (2018), Meier, Ray-Mermet, and Rothen (2015), Ward et al (2013), and one year later, even Rothen and Meier (2010) themselves, all found some sort of memory advantage in adult synesthetes with a variety of synesthesia types, suggesting that, despite Rother and Meier's (2009) findings, synesthesia often attributes to enhanced memory.

However, the question remains: How does this memory advantage develop with age? All of the above studies were performed on young adults, with little variation of participant age. It has recently been discovered that synesthesia gets stronger throughout childhood (Simner \& Bain, 2013) and diminishes with older age (Simner et al, 2017), so when, exactly, does synesthesia's benefit to memory begin to manifest?

Green and Goswami (2008) attempted to answer this question with 10 grapheme-color synesthetes ages 715. The children were given a matrix memory task, in which they were shown a grid of 16 boxes with numbers in three trials (one with black numbers, one with colors consistent with concurrents, and one with colors inconsistent with concurrents) and were asked to fill in the grid immediately after viewing. They concluded that synesthetic children do not have enhanced memory over their peers, possibly because of their low education levels.

However, Simner and Bain (2018) refute the validity of Green and Goswami's study because the researchers do not describe their method of recruiting participants, other than the phrase, "Six grapheme-color synesthetes, four girls and two boys, took part in the study." The lack of clarity in this description leads the reader to assume that the researchers received their participants from parents involved in the scientific community who were actively aware of their child's synesthesia. This means that the child was likely to be overly aware of their synesthesia, which increases synesthesia's development compared to typical synesthetes in this age range. For these reasons, the results of this study cannot be considered representative of the typical child synesthete. Simner and Bain instead used random-population testing to remedy this issue, identifying five grapheme-color synesthetes ages 10-11 with mass-scale MRI testing of over 600 children. Despite this change in recruitment style, Simner and Bain found similar results to Green and 
Goswami upon replicating their research, concluding that, while child synesthetes perform better in some areas, their synesthesia is simply too underdeveloped to produce significant benefits.

However, both of these studies left out a key age group: teenagers. It is apparent that synesthesia does eventually induce advantageous memory, as this has been shown in group studies using young adult synesthetes. It is also apparent that this advantage does not appear in children, as this has been shown in group studies using child synesthetes. Currently, there is only one age group that has not been studied explicitly or exclusively for a memory advantage related to synesthesia: ages 13-18. Because of the lack of research, it is unclear whether teenage synesthetes have advantageous memory, like their older counterparts, or have yet to develop it, like their younger counterparts. Simner and Bain (2018) suggested that their study be repeated in an educational setting, to determine the effects of higher level (in this case, high school versus elementary school) education on synesthesia and memory development. In this paper, the intent was to do just this: replicate Simner and Bain's method on a group of teenage synesthetes and controls from Wekiva High School, a typical American secondary school, to determine whether teenage synesthetes present benefited memory over their non-synesthetic peers.

\section{Materials and Method}

\section{Participant Collection}

In order to collect synesthetes and controls that are representative of the teenage synesthete and non-synesthete populations, participants must be randomly selected from a sample population and must not be aware of the synesthesia aspect of the study in which they are participating (Simner and Bain, 2018). In this case, the sample population was the student body of Wekiva High School, and random selection occurred by random survey sampling. A link to a Google Form survey including questions taken directly from the Synesthesia Battery's "Find out if you might have Synesthesia" quiz was written on various classroom whiteboards around the school campus to ensure randomness. The full list of questions used includes:

Do numbers or letters cause you to have a color experience? Example: Does the letter J "mean" yellow to you? Or does "5" make you perceive purple?

Do weekdays and months have specific colors? Example: Does July always mean Navy Blue to you? Is Wednesday always orange?

Do you imagine or visualize weekdays, months and/or years as having a particular location in space around you? Example: Is September always located two feet in front of you to the left?

Does hearing a sound make you perceive a color? Example: Does a shrill car horn cause you to see the color green? Does $\mathrm{C}$ sharp make you see pink? Does a specific song sound orange?

Do certain words trigger a taste in your mouth? Example: Does the name 'Max' taste like earwax?

Do you feel a sense of touch when you smell things? Example: Does the smell of coffee make you feel as though you are touching a cold glass surface?

Do you suspect that you experience an unusual blending that other people do not have (other than the ones listed above)? These could include automatically hearing a sound when you see movement, or the sense of a shape being triggered by a taste, or experiencing a color when feeling pain. If so, please describe your experiences here.

Before beginning the survey, all participants gave their informed consent. Participants were given the option to answer the questions with "yes," or "no." There were 90 total responses to the survey. All respondents who answered "yes" on one or more questions were contacted about further participation. The first 50 respondents who answered "no" on all questions were contacted about further participation 


\section{Confirming Synesthesia}

A "golden standard" for diagnosing grapheme-color synesthesia is through consistency testing, which is why the Synesthesia Battery is considered so reliable. However, the Synesthesia Battery has not yet been made available to test a broad range of synesthesia types. Also, due to a policy change in December 2019, the Synesthesia Battery is no longer available for use to those under the age of 18. As I am under 18, I was unable to secure this testing for study. In order to confirm if participants who indicated synesthetic experiences on the survey described above were actually synesthetes, a simplified copy of Rouw and Scholte's (2016) synesthesia confirmation test was created using Google Forms. Upon being determined fit for the study, participants were emailed a link to the confirmation test form. Participants were given a statement followed by a Likert Scale in which they were asked to rate how the statement applied to them on a scale of one to five, with one being "not at all" and five being "all the time." A statement relating to all five of the synesthesia clusters mentioned above (Novich et al, 2011) was given in the test. The statements used include "Letters, numbers, days, months, or years cause me to experience specific colors," "Music, the sounds of musical instruments, tones, or pitches cause me to experience specific colors/patterns," "Pain, personality type, emotion, temperature, touch on the skin, taste in the mouth, or scents cause me to experience specific colors," "I see years, months, numbers, or letters in a 3D shape, in a line, or in a certain location in space around me," and "Sounds make me experience certain smells or tastes, or give me a touch/sensation on the skin." If a participant selected three or above on the Likert Scale, they were further prompted to give three extremely specific examples of their experiences. Next, the provided answers were classified into one of 4 categories: incomplete answer, in which there was not enough information to grade the example otherwise (e.g, "red" rather than "A is red"), too few examples, in which less than 3 were given, bad examples, and good examples. Classification for the last two categories was based on the very strict criteria of Rouw and Scholte (2016). The first criterion was specific inducers and concurrents. Examples were "bad" if the inducer was not specific (e.g, "everything" rather than "numbers"), or if the concurrents caused by the inducer were not specific (e.g, "a feeling" rather than "a tingling sensation"). The second criterion was common associations. Examples were "bad" if they were cross-modality (e.g, "the sound of pouring coffee makes me smell coffee") or were consistently analogous to the inducer for all inducers (e.g, " $\mathrm{r}$ is red because 'red' starts with r", "w is white because 'white' starts with w"). Lastly, common sensations were considered "bad" (e.g, "the sound of nails on a chalkboard makes me shiver"). Examples of a "good" response would be "I see the letter A as red in my head," and, "Whenever I hear jazz music, I see the color orange." If a participant provided three examples classified as "good", they were included in the study as a synesthete. If a participant provided only "bad" examples or less than three "good" examples, they were excluded from the study to prevent skewing the data with "weak" synesthesia. Finally, if a participant selected two or less on the Likert Scale for every question and could provide zero examples whatsoever, they were included as a non-synesthetic control.

\section{Participants}

Table 1. All participant age, grade level, and grouping information

\begin{tabular}{|l|l|l|l|}
\hline Classification Initial & Age & Grade Level & Grouping \\
\hline W & 18 & 12 th & Control \\
\hline S & 14 & 9 th & Control \\
\hline P & 17 & 12 th & Control \\
\hline N & 17 & 11 th & Control \\
\hline
\end{tabular}




\begin{tabular}{|c|c|c|c|}
\hline G & 16 & 11th & Control \\
\hline $\mathrm{L}$ & 17 & 11 th & Control \\
\hline $\mathrm{R}$ & 15 & 10th & Control \\
\hline G2 & 18 & 12th & Control \\
\hline $\mathrm{F}$ & 16 & 11th & Control \\
\hline S2 & 17 & 12 th & Control \\
\hline B & 16 & 10th & Control \\
\hline R2 & 17 & 11 th & Control \\
\hline \multicolumn{4}{|l|}{ Table 1. continued.... } \\
\hline Classification Initial & Age & Grade Level & Grouping \\
\hline W2 & 16 & 11 th & Control \\
\hline R3 & 16 & 11 th & Control \\
\hline $\mathrm{F} 2$ & 17 & 11 th & Control \\
\hline $\mathrm{N} 2$ & 18 & 12 th & Control \\
\hline R4 & 15 & 10th & Control \\
\hline R5 & 16 & 10th & Control \\
\hline $\mathrm{C}$ & 16 & 11 th & Synesthete \\
\hline $\mathrm{D}$ & 15 & 10th & Synesthete \\
\hline $\mathrm{U}$ & 18 & 12 th & Synesthete \\
\hline M & 17 & 11 th & Synesthete \\
\hline
\end{tabular}

Four synesthetes of varying ages were selected for participation. This sample size matches that of Green and Goswami (2008) and Simner and Bain (2018). 18 controls of varying ages were selected for participation. This is lower than that of the previously mentioned studies because, of the 50 students emailed, only 18 were found to be true controls and were willing to participate further. Due to Green and Goswami not mentioning their collection method, and Simner and Bain utilizing MRI scanning on over 600 children, I was unable to replicate this collection method because, as a high school student, I do not have access to an MRI machine or hundreds of willing teenagers. However, many studies, including Robinson (2015), Rothen and Meier (2009), Smilek (2002), and Witthoft and Winawer (2015) have similar sample sizes to the one used in this study and were able to generate conclusive results from their data. Therefore, this study's sample size should not be of concern. 


\section{Tests for Memory Advantage}

All participants were asked to stay after school on a specific day to complete the memory tests. All tests were modeled after Simner and Bain (2018). The matrix used during testing is located in Figure 1. All data was collected in a Microsoft Excel spreadsheet.

\section{Immediate Recall}

Participants were shown a string of 15 non-repeated mixed graphemes (both numbers and letters) on printed paper and were given 30 seconds to study it. Graphemes were in 32-point Times New Roman font. After 30 seconds, the paper was taken away, a new, blank paper was given, and participants were asked to write down as much of the string as they remembered on their own paper. This was done twice with two different strings. The string used for Immediate Recall 1 was 57AI9EGMQ382KG4. The string used for Immediate Recall 2 was ZEJ089D7NTXKR4P. Data was collected by number of correct graphemes recalled and was classified individually and by grouping (synesthete, nonsynesthete).

\section{Delayed Recall}

Participants were shown a new string of 15 non-repeated mixed graphemes and were given 1 minute and 30 seconds to study it. Graphemes were in 32-point Times New Roman font. After 1 minute and 30 seconds, the paper was taken away, a new, blank paper was given, and participants were instructed to wait 30 minutes. After 30 minutes, they were asked to write down as much of the string as they remembered on their own paper. The string used for this test was 1VMBW9IQN5BCK6Y. Data was collected by number of correct graphemes recalled and was classified individually and by grouping (synesthete, non-synesthete).

\section{Matrix Recall}

A 16-cell, 4 by 4 square matrix was presented on paper. The squares were filled with non-repeated mixed graphemes in 60-point Times New Roman font and were centered in the cell. Cells were 1.5 by 1.5 inches each. Participants were given 30 seconds to study the matrix, after which it was taken away. Participants were then given a blank paper copy of the matrix and were instructed to fill it in to the best of their ability. Data was collected by number of correct graphemes recalled and was classified individually and by grouping (synesthete, non-synesthete).

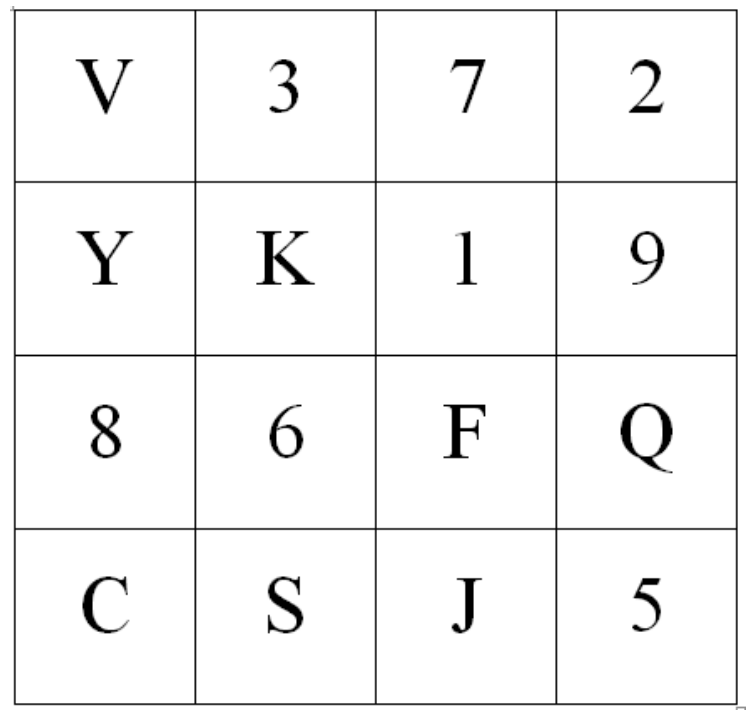

Figure 1. Not-to-scale matrix used for Matrix Recall 


\section{Calculations and Statistics}

\section{Individual}

For each test, the standard deviation and mean of the control scores was taken using Microsoft Excel, and synesthete scores were compared. Synesthetes were considered to have a memory advantage (however small) if their raw scores were more than one standard deviation above the control mean. This method of calculation and memory-advantage determination was used per Rothen and Meier (2009).

\section{Group}

For each test, results were put through a Two Proportion T-test on Microsoft Excel. The two groups for this test were synesthetes and non-synesthetes. This test was used because both Green and Goswami (2008) and Simner and Bain (2018) used an ANOVA. However, ANOVAs are most optimal only if there are three groups to calculate between. Since a T-test and ANOVA are similar for comparing significance, and a T-test uses two groups, a T-test was determined to be the best option for this study. Per Levene's test, the findings were considered statistically significant if the resulting calculation was greater than .05 .

\section{Results}

Table 2. Raw data (correct/incorrect answers) for each participant in each test

\begin{tabular}{|l|l|l|l|l|}
\hline $\begin{array}{l}\text { Classification Ini- } \\
\text { tial }\end{array}$ & Immediate 1 \# Correct & Immediate 2 \# Correct & Delayed \# Correct & $\begin{array}{l}\text { Matrix \# Cor- } \\
\text { rect }\end{array}$ \\
\hline C & 10 & 14 & 7 & 11 \\
\hline U & 8 & 13 & 8 & 11 \\
\hline M & 11 & 15 & 14 & 13 \\
\hline D & 11 & 8 & 9 & 9 \\
\hline W & 10 & 9 & 8 & 9 \\
\hline S & 9 & 10 & 10 & 3 \\
\hline P & 8 & 9 & 7 & 8 \\
\hline N & 9 & 11 & 6 & 11 \\
\hline G & 9 & 4 & 8 & 7 \\
\hline L & 7 & 7 & 9 & 8 \\
\hline R & 6 & 5 & 14 & 6 \\
\hline G2 & 7 & 9 & 7 & 4 \\
\hline
\end{tabular}




\begin{tabular}{|l|l|l|l|l|}
\hline \multicolumn{2}{|l|}{ Table 2. continued... } \\
\hline $\begin{array}{l}\text { Classification Ini- } \\
\text { tial }\end{array}$ & Immediate 1 \# Correct & Immediate 2 \# Correct & Delayed \# Correct & $\begin{array}{l}\text { Matrix \# Cor- } \\
\text { rect }\end{array}$ \\
\hline F & 9 & 12 & 15 & 14 \\
\hline S2 & 9 & 9 & 10 & 8 \\
\hline B & 10 & 9 & 9 & 14 \\
\hline R2 & 8 & 9 & 12 & 6 \\
\hline W2 & 8 & 7 & 5 & 5 \\
\hline R3 & 11 & 9 & 14 & 7 \\
\hline F2 & 11 & 7 & 7 & 10 \\
\hline N2 & 12 & 9 & 13 & 9 \\
\hline R4 & 8 & 6 & 5 & 7 \\
\hline R5 & 10 & 10 & 14 & 10 \\
\hline
\end{tabular}

\section{Individual Measurement}

For all individual calculations, the mean and standard deviation of the control group for each test was compared to individual synesthete performance for the corresponding test. Synesthetes were considered to have a memory advantage if they performed more than one standard deviation above the control group mean. For Immediate Recall 1 $\left(\mathrm{x}^{-}=8.94, \mathrm{SD}=1.5\right)$, synesthetes $\mathrm{M}$ and $\mathrm{D}$ showed statistical memory advantage, while synesthetes $\mathrm{U}$ and $\mathrm{C}$ did not. For Immediate Recall $2\left(\mathrm{x}^{-}=8.44, \mathrm{SD}=2.1\right)$, synesthetes $\mathrm{C}, \mathrm{U}$, and $\mathrm{M}$ showed statistical memory advantage, while synesthete $\mathrm{D}$ did not. For Delayed Recall $\left(\mathrm{x}^{-}=9.61, \mathrm{SD}=3.3\right)$, only synesthete $\mathrm{M}$ showed statistical memory advantage. For Matrix Recall $\left(\mathrm{x}^{-}=8.11, \mathrm{SD}=2.98\right)$ only synesthete $\mathrm{M}$ showed statistical memory advantage.

Table 3. Means, standard deviation, and synesthete indication of memory advantage (+/-)

\begin{tabular}{|l|l|l|l|l|l|l|}
\hline Test & Mean & Standard Deviation & Syn. C & Syn. U & Syn. M & Syn. D \\
\hline I1 & 8.94 & 1.5 & - & - & + & + \\
\hline I2 & 8.44 & 2.1 & + & + & + & - \\
\hline D & 9.61 & 3.3 & - & - & + & - \\
\hline M & 8.11 & 2.98 & - & - & + & - \\
\hline
\end{tabular}




\section{Group Measurement}

\section{Immediate Recall 1}

In this task, participants immediately recalled (in writing) a string of 15 graphemes shown on paper. No statistically significant memory advantage was found for synesthetes over controls when a two-variable T-test was conducted at the $95 \%$ confidence level $(\mathrm{T}=.24, \mathrm{p}<.05)$.

\section{Immediate Recall 2}

In this task, participants performed the same procedure as Immediate Recall 1 with a different string of graphemes. No statistically significant memory advantage was found for synesthetes over controls using the two-variable T-test. However, the value was approaching significance $(\mathrm{T}=.06, \mathrm{p}<.05)$.

\section{Delayed Recall}

In this task, participants recalled (in writing) a string of 15 graphemes shown on paper after 30 minutes of delay from the initial time of memorization. No statistically significant memory advantage was found for synesthetes over controls by the two-variable $\mathrm{T}$-test $(\mathrm{T}=.95, \mathrm{p}<.05)$.

\section{Matrix Recall}

In this task, participants immediately recalled a 4 by 4 square matrix with a grapheme in each square. The matrix recall was the only group test to show a statistically significant memory advantage $(\mathrm{T}=.027, \mathrm{p}<.05)$.

\section{Discussion and Conclusion}

\section{Individual}

For all individual tests, at least one synesthete performed at a level of memory advantage compared to the average performance of the controls. This is in line with the results of Rothen and Meier (2009), in which their synesthetes performed at a level of significance individually but not as a group. Rothen and Meier attribute this difference between individual and group performance, for all immediate, delayed, and matrix tests, to individual memory differences independent of synesthesia. They also concluded that individual case studies that found statistical significance, such as Smilek (2002), did so due to that specific synesthete's purposeful strategic use of synesthesia to assist them in remembering. Because the synesthetes in this study were not told that they were classified as synesthetes, or even that this study involved the testing of synesthesia, they could not have known to use their synesthesia strategically. Additionally, even if they had used their synesthesia strategically, it did not give them a consistent advantage over those without synesthesia. Therefore, the statistical advantages in memory abilities found for some synesthetes in certain tests cannot be only or entirely accredited to the presence of synesthesia because not all synesthetes showed an advantage for any one test, and no one synesthete showed an advantage for all tests. Rather, individual differences in general memory ability could account for the differences in performance between synesthetes and controls, as well as between synesthetes. Thus, the supposed individual memory advantages found for some synesthetes should be mostly connected to their individual general memory abilities instead of to their synesthesia or synesthetic memory. Since this trend was found in both the adults of Rothen and Meier (2009) and the teenagers of this study, it can be concluded that age difference between adulthood and adolescence makes little difference in individual memory advantage for synesthetes versus controls. 


\section{Group}

For group testing, 3 of the 4 tests (immediate recall 1, immediate recall 2, and delayed recall) did not show a statistically significant difference in memory abilities between synesthetes and controls. Again, this is in line with the findings of Rothen and Meier (2009) in that individual synesthetes showed some advantage, but the group overall proved no statistical advantage. Rothen and Meier additionally credit this to individual differences between general memory abilities of synesthetes and controls. Since no test or classification was done for general memory abilities in Rothen and Meier (2009) or this study, it is unknown whether the controls or the synesthetes in either study had above- or below-average general memory. Thus, it will remain unknown whether there were substantial differences in general memory abilities between controls and synesthetes that could have accounted for the lack of statistical significance. The findings in this study are also in line with those of Simner and Bain (2018), who found no statistical advantage in their group of child synesthetes. However, Simner and Bain consider the lack of significance to be because of the low levels of education in their participants, who were in elementary school. Because the synesthetes in this study had received at least four more years of education than all of the participants in Simner and Bain (2018), but still did not perform at a level of significance, level of education, from primary to secondary schooling, cannot be considered a factor in the lack of memory advantage in child or adolescent synesthetes. Therefore, the lack of a memory advantage for the immediate and delayed recall tests should be attributed to the differences in general memory between synesthetes and controls, and not to synesthetic memory advantage or lack thereof.

The matrix recall was the only test that showed a group memory advantage for synesthetes over controls. Given that none of the synesthetes used in this study were sequence-space synesthetes, who have been shown to have increased spatial memory (Brang 2013), these results are surprising. However, the results of the matrix recall from Simner and Bain (2018), though not significant, were approaching and came extremely close to significance, indicating that child grapheme-color, not sequence-space, synesthetes were very close to having a memory advantage in matrix memory. Green and Goswami (2008) also found results for their matrix task that mirror those of Simner and Bain (2018). Therefore, because the results of this study indicate significance in teenagers while previous studies have shown results approaching significance in children, it can be concluded that a memory advantage for matrix recall in synesthetes develops sometime between childhood and adolescence. One possible reason for this is that older age allows for longer experience with synesthesia. One conclusion of Simner and Bain (2018) was that they found no significance because their participants' synesthesia was simply not developed enough due to their young age. As the synesthetes in this study were older than Simner and Bain's and had had more time for their synesthesia to develop, it is possible that their synesthesia had matured enough to allow a memory advantage in adolescence where it was not present in childhood. According to Simner et al (2017), synesthesia ages with the synesthete and develops into maximum strength during young adulthood. Since the synesthetes in this study were approaching young adulthood, it is plausible that their synesthesia had matured, or strengthened, enough to provide a change from no advantage to advantage in matrix recall memory.

\section{Limitations}

From the results of this study, it can be concluded that teenage synesthetes present an advantage in matrix memory over non-synesthetes. However, there are several limitations to this study that should be examined in future research. Firstly, the type of synesthesia in this study was not controlled for due to being limited to the population of one school and the rarity of synesthesia. Had synesthesia type (e.g, grapheme-color) been controlled for, it was likely that not enough synesthetes would have been found for participation. Nevertheless, most studies of similar scope control for grapheme-color synesthesia as it allows the researchers to perform a color congruency memory test in which grapheme strings are presented not just in black and white, but in colors congruent and incongruent with participants' synesthesia. This test allows a clearer conclusion over the effects of synesthesia on memory. Because this study did not control for synesthesia type, no test using color congruency could be performed, meaning there was less data involving 
synesthetic memory and more involving general memory. Future studies involving teenage synesthetes and memory abilities should be sure to control for the type of synesthesia in participants in order to be able to make a stronger conclusion about synesthetic memory.

Secondly, this study did not utilize the Synesthesia Battery for synesthesia confirmation, which is known and credited as being the "gold standard" for synesthesia testing. This test was not used because I, the researcher, do not reach the age minimum of 18 years required to use the Battery. Upon contacting the creators of the Battery about using the software despite being under the age limit, I received no response. Because the Synesthesia Battery was not available, I used the less-credited synesthesia confirmation test by Rouw and Sholte (2016). Although this test is able to confirm synesthesia, it is less reliable than the Synesthesia Battery because it requires the researcher's personal interpretation of the results. Therefore, the synesthetes in this study may have had weaker synesthesia than synesthetes in other studies because it is quite possible that $\mathrm{I}$, as an inexperienced researcher, made a few mistakes in classification. Stronger or weaker synesthesia could be the difference between stronger and weaker memory. The Synesthesia Battery eliminates this possibility of human error. Future researchers should be sure to secure the use of the Synesthesia Battery before beginning testing.

Lastly, no assessment of participant general memory abilities was performed in this study. Simner and Bain (2018) performed a general memory assessment and classified their controls as either high or low memory prior to doing the synesthetic memory tests in order to have a higher degree of certainty in their conclusion that no memory advantage was found. Due to this specific study being performed on high school students by a high school student, all testing had to be done after school in order to ensure participation. There was limited time after school due to transportation issues for some participants (e.g, a person who rides the school bus much catch the school bus before it leaves, and therefore has little extra time after school), and there are no reliable and conclusive general memory tests that are accessible or short enough to be done in a small time frame. In this study, it remains unknown whether the controls had low, average, or high memory, which all could have affected their performance on the memory tests given. Having a control with above or below average memory could result in an outlier in the data, which could skew the standard deviation and mean of the control group and make it either easier or harder for a synesthete to achieve the significance level that indicates memory advantage. Future studies should always perform a general memory test on both controls and synesthetes to ensure that their controls do not produce outliers that could skew the data and the interpretations of it.

\section{References}

Banissy, M. J., et al. (2013). Personality traits in people with synaesthesia: Do synaesthetes have an atypical personality profile? Personality and Individual Differences, 54(7), 828-831.

https://www.sciencedirect.com/science/article/abs/pii/S0191886912006289

Bankieris, K., Aslin, R. (2016). Explicit associative learning and memory in synesthetes and non synesthetes.i-Perception, 7(5), 1-12.: :/journals.sagepub.com/doi/pdf/10.1177/2041669516658488

Brang, D., Miller, L. E., McQuire, M., Ramachandran, V. S., Coulson, S. (2013). Enhanced mental rotation ability in time-space synesthesia. Cognitive Processing, 14(4), 429-434.

https://www.researchgate.net/profile/David Brang2/publication/236104266

Chun, C. A., Hupé, J. (2015). Are synesthetes exceptional beyond their synesthetic associations? A systematic comparison of creativity, personality, cognition, and mental imagery in synesthetes and controls. British Journal of Psychology, 107(3), 397-418.

https://onlinelibrary.wiley.com/doi/pdf/10.1111/bjop.12146 
Eagleman, D. M., et al. (2007). A standardized test battery for the study of synesthesia. Journal of Neuroscience Methods 159(1), 139-145.

https://www.sciencedirect.com/science/article/pii/S0165027006003153

Green, J. A. K., \& Goswami, U. (2008). Synaesthesia and number cognition in children. Cognition, 106,463-473. https://www.cne.psychol.cam.ac.uk/pdfs/publication-pdfs/COGNIT1632 2007.pdf

Gross, V., Neargarder, S. (2011). Superior encoding enhances recall in color-graphemic synesthesia. Perception, 40(2), 196-208. https://www.researchgate.net/publication/51199

Hale, J., Thompson, J. M., Morgan, H. M., Cappelletti, M., \& Cohen Kadosh, R. (2014). Better together? The cognitive advantages of synaesthesia for time, numbers, and space.

Cognitive Neuropsychology, 31(7/8), 545-564.https://doi.org/10.1080/02643294.2014.96

Hanggi, J., et al. (2011). Globally Altered Structural Brain Topology in Grapheme-Color Synesthesia. The Journal of Neuroscience, 31(15), 5816-5828.

https://www.jneurosci.org/content/jneuro/31/15/5816.full.pdf

Kadosh, R., Gertner, L., Terhune, D. (2011). Exceptional Abilities in the Spatial Representation of Numbers and Time: Insights from Synesthesia. The Neuroscientist, 18(3), 208-215.

https://eprints.goldsmiths.ac.uk/17076/1/Cohen\%

Lunke, K., \& Meier, B. (2018). New insights into mechanisms of enhanced synaesthetic memory: Benefits are synaesthesia-type-specific. PLoS ONE, 13(9), 1-17.

https://doi.org/10.1371/journal.pone.0203055

Luria, A. R. (1968). The Mind of a Mneumonist: A Little Book About a Vast Memory.

https://science.sciencemag.org/content/161/3839/349

Meier, B., Rothen, N. (2013). Grapheme-color synaesthesia is associated with a distinct cognitive style. Frontiers in Psychology, 4(632). file://C:/Users/4804247243/Downloads/Grapheme-color_synaesthesia_is_associate.pdf

Meier, B., Rey-Mermet, A., \& Rothen, N. (2015). Turning univalent stimuli bivalent: Synesthesia can cause cognitive conflict in task switching. Cognitive Neuroscience, 6(2/3), 48-55.

https://doi.org/10.1080/17588928.2015.1017449

Novich, S., Cheng, S., Eagleman, D. M. (2011). Is synaesthesia one condition or many? A large-scale analysis reveals subgroups. Journal of Neuropsychopathy, 5(2), 353-371.

http://hstrial-tridenttechnical.homestead.com/NovichChengEagleman

Pfeifer, G., et al. (2014). Associative memory advantage in grapheme-color synesthetes compared to older, but not young adults. Frontiers in Psychology.

https://www.frontiersin.org/articles/10.3389/fpsyg.2014.00696/full

Pfeifer, G., Ward, J., Chan, D., \& Sigala, N. (2016). Representational Account of Memory: Insights from Aging and Synesthesia. Journal of Cognitive Neuroscience, 28(12), 1987-2002. https://doi.org/10.1162/wjocn a 01014 
Radvansky, G., \& Gibson, B. (2011). Synesthesia and Memory: Color Congruency, von Restorff, and False Memory Effects. Journal of Experimental Psychology Learning Memory and Cognition, 37(1), 219-229.

https://www.researchgate.net/publication/4976

Robinson, C. (2015). Synesthesia and Memory: An Exploratory Analysis. Modern Psychological Studies,21(1), 8493.http://search.ebscohost.com/login.aspx?direct=true \&db=a9h\&

Rothen, N., et al., (2012). Enhanced memory ability: Insights from synaesthesia..Neurosci.Biobehav. Rev. http://dx.doi.org/10.1016/j.neubiorev.2012.05.004

Rothen, N., Meier, B. (2009). Do Synesthetes Have a General Advantage in Visual Search and Episodic Memory? A Case for Group Studies. PLoS ONE 4(4): e5037.

https://doi.org/10.1371/journal.pone.0005037

Rothen, N., Meier, B. (2010). Grapheme-colour synaesthesia yields an ordinary rather than extraordinary memory advantage: Evidence from a group study. Memory, 18(3), 258-264

https://doi.org/10.1080/09658210903527308

Rouw, R., Scholte, H.S., (2016). Personality and cognitive profiles of a general synesthetic trait. Neuropsychologia, 88, 35-48. http://dx.doi.org/10.1016/j.neuropsychologia.2016.01.006j

Simner, J., Bain, A. E. (2013).A longitudinal study of grapheme-color synesthesia in childhood: 6/7 years to 10/11 years. Frontiers in Psychology.

https://www.frontiersin.org/articles/10.3389/fnhum.2013.00603/ful

Simner, J., Bain, A. E. (2018). Do children with grapheme-colour synaesthesia show cognitive benefits? British Journal of Psychology, 109(1), 118-136.

Retrieved from https://doi.org/10.1111/bjop.12248

Simner, J., Ipser, A., Smees, R., Alvarez, J. (2017). Does synaesthesia age? Changes in the quality and consistency of synaesthetic associations. Neuropsychologia, 106, 407-416.

https://www.sciencedirect.com/science/article/abs/pii/S002839321730

Smees, R., Hughes, J., Carmichael, D. A., Simner, J. (2019). Learning in colour: children with grapheme-colour synaesthesia show cognitive benefits in vocabulary and self-evaluated reading. Philosophical Transactions of the Royal Society B, 374(1787).

https://royalsocietypublishing.org/doi/full/10.1098/rstb.2018.0348

Smilek, D., Dixon, M., Cudahy, C. (2002). Synesthetic color experiences influence memory. Psychological Science, 13(6), 548-552.

http://pcl.missouri.edu/jeff/sites/pcl.missouri.edu.jeff/files/smilek.pdf

Ward, J., Hovard, P., Jones, A., Rothen, N. (2013). Enhanced recognition memory in grapheme-color synaesthesia for different categories of visual stimuli. Frontiers in

Psychology, 4(762). https://www.frontiersin.org/articles/10.3389/fpsyg.2013.00762/full 
Witthoft, N., \& Winawer, J. (2013). Learning, memory, and synesthesia. Psychological science, 24(3), 258-265. https://www.ncbi.nlm.nih.gov/pmc/articles/PMC3648671/ 